Yukawa Institute Kyoto

YITP-04-21

hep-th/0404089

April 2004

\title{
Central elements of the elliptic $\mathbb{Z}_{n}$ monodromy matrix algebra at roots of unity
}

\author{
Wen-Li Yang $^{a, b} \quad$ Alexander Belavin $^{c}$ and Ryu Sasaki ${ }^{d}$ \\ ${ }^{a}$ Institute of Modern Physics, Northwest University Xian 710069, P. R. China \\ ${ }^{b}$ Department of Mathematics, University of Queensland, Brisbane, QLD 4072, Australia \\ ${ }^{c}$ Landau Institute for Theoretical Physics, Chernogolovka, 142432, Russia \\ ${ }^{d}$ Yukawa Institute for Theoretical Physics, \\ Kyoto University, Kyoto 606-8502, Japan
}

\begin{abstract}
The central elements of the algebra of monodromy matrices associated with the $\mathbb{Z}_{n}$ R-matrix are studied. When the crossing parameter $w$ takes a special rational value $w=\frac{n}{N}$, where $N$ and $n$ are positive coprime integers, the center is substantially larger than that in the generic case for which the "quantum determinant" provides the center. In the trigonometric limit, the situation corresponds to the quantum group at roots of unity. This is a higher rank generalization of the recent results by Belavin and Jimbo.
\end{abstract}

PACS: 03.65.Fd; 05.30.-d

Keywords: Integrable models; Elliptical quantum goup and quantum group; Central elements. 


\section{Introduction}

The algebra of the monodromy matrix (or Yang-Baxter algebra) is generated by noncommutative matrix elements of the L-operator $L(u)$ satisfying a quadratic "RLL" relation

$$
R_{12}(u-v) L_{1}(u) L_{2}(v)=L_{2}(v) L_{1}(u) R_{12}(u-v),
$$

where $L_{1}(u)=L(u) \otimes 1, L_{2}(u)=1 \otimes L(u)$ and $R(u)$ is the R-matrix, a solution of quantum Yang-Baxter equation

$$
R_{12}(u-v) R_{13}(u) R_{23}(v)=R_{23}(v) R_{13}(u) R_{12}(u-v) .
$$

We adopt the standard notation: $R_{i j}(u)$ is an embedding operator of R-matrix in the tensor space $\mathbb{C}^{n} \otimes \mathbb{C}^{n} \cdots$, which acts as identity on the factor spaces except for the $i$-th and $j$-th ones. If $R(u)$ is a rational R-matrix, the algebra after a proper specialization is a Yangian algebra [1]. For a trigonometric R-matrix, the algebra is closely connected with a quantum group [2, 3]. If $R(u)$ is an elliptic R-matrix, the algebra gives rise to a Sklyanin algebra [4, 5].

In this paper, we will address the issue of the center of an elliptic algebra associated with the $\mathbb{Z}_{n}$ R-matrix [6] when the crossing parameter takes rational values. In the trigonometric limit, it corresponds to a quantum group at roots of unity. So here we call this case the "roots of unity " case as its trigonometric limit. As is well known, for the trigonometric algebra (as a degenerate algebra of the elliptic algebra) when the crossing parameter (deformation parameter) is in roots of unity case the center is much larger than that of the generic case [7, 8. Recently for the simplest case of the $\mathbb{Z}_{2}$ R-matrix (or eight-vertex R-matrix) 9], the structure of the center in the "roots of unity" case was clarified [10]. Here, we will consider the question with a generic case $n \geq 2$.

The paper is organized as follows. In section 2 we give the definition of the elliptic algebra and introduce appropriate notation. We formulate the fundamental Boltzmann weights of the $A_{n-1}$ type interaction-around-a-face (IRF) model (or the SOS model) and give the corresponding face-vertex correspondence relation in section 3. In section 4, we construct the symmetric fusion procedure for the R-matrices, Boltzmann weights and intertwining vectors, which are useful to handle the center of the algebra. Finally, we give the center of the elliptic

algebra with the $\mathbb{Z}_{n}$ R-matrix when the crossing parameter is in the "roots of unity" case in section 5 . Section 6 is for conclusions. 


\section{The Algebra of Monodromy Matrices}

Let us fix an integer $n(n \geqslant 2)$ and a complex number $\tau$ such that $\operatorname{Im}(\tau)>0$. Introduce the following elliptic functions

$$
\begin{aligned}
& \theta\left[\begin{array}{l}
a \\
b
\end{array}\right](u, \tau)=\sum_{m=-\infty}^{\infty} \exp \left\{\sqrt{-1} \pi\left[(m+a)^{2} \tau+2(m+a)(u+b)\right]\right\} \\
& \theta^{(j)}(u)=\theta\left[\begin{array}{c}
\frac{1}{2}-\frac{j}{n} \\
\frac{1}{2}
\end{array}\right](u, n \tau), \quad \sigma(u)=\theta\left[\begin{array}{c}
\frac{1}{2} \\
\frac{1}{2}
\end{array}\right](u, \tau), \\
& \bar{\theta}^{(j)}(u)=\exp \{\sqrt{-1} \pi u\} \theta^{(j)}(u) .
\end{aligned}
$$

Among them the $\sigma$-function ${ }^{1}$ satisfies the following identity:

$$
\begin{aligned}
& \sigma(u+x) \sigma(u-x) \sigma(v+y) \sigma(v-y)-\sigma(u+y) \sigma(u-y) \sigma(v+x) \sigma(v-x) \\
& \quad=\sigma(u+v) \sigma(u-v) \sigma(x+y) \sigma(x-y) .
\end{aligned}
$$

Let $\left\{e_{i} \mid i=1,2, \cdots, n\right\}$ be the orthonormal basis of the vector space $\mathbb{C}^{n}$ such that $\left\langle e_{i}, e_{j}\right\rangle=\delta_{i j}$. Let $R(u) \in \operatorname{End}\left(\mathbb{C}^{n} \otimes \mathbb{C}^{n}\right)$ be the $\mathbb{Z}_{n}$ R-matrix given by

$$
R(u)=\sum_{i, j, k, l} R_{i j}^{k l}(u) E_{i k} \otimes E_{l j},
$$

in which $E_{i j}$ is the matrix with elements $\left(E_{i j}\right)_{k}^{l}=\delta_{j k} \delta_{i l}$. The coefficient functions are [12, 13]

$$
R_{i j}^{k l}(u)= \begin{cases}\frac{h(u) \theta^{(i-j)}(u+w)}{\theta^{(i-k)}(w) \theta^{(k-j)}(u)} & \text { if } i+j=k+l \bmod n \\ 0 & \text { otherwise }\end{cases}
$$

where a complex parameter $w$ is called the crossing parameter. We have set

$$
h(u)=\frac{\prod_{j=0}^{n-1} \theta^{(j)}(u)}{\prod_{j=1}^{n-1} \theta^{(j)}(0)} .
$$

The R-matrix satisfies the quantum Yang-Baxter equation (1.2), unitarity and crossingunitarity relations [12. It should be remarked that our R-matrix given in (2.4) and (2.5) is the same as that of Ref.[13], and that our R-matrix $R(u)$ is related to the R-matrix $S(u)$ of Richey and Tracy [12] by $R_{12}(u)=\frac{e^{\sqrt{-1} \pi u}}{n} S_{12}^{t_{1} t_{2}}(u)$, where $t_{i}$ denotes the transposition in the $i$-th space. Consequently, the R-matrix enjoys the following property:

\footnotetext{
${ }^{1}$ Our $\sigma$-function is the $\vartheta$-function $\vartheta_{1}(u)$ 11. It has the following relation with the Weierstrassian $\sigma$ function if denoted it by $\sigma_{w}(u): \sigma_{w}(u) \propto e^{\eta_{1} u^{2}} \sigma(u), \eta_{1}=\pi^{2}\left(\frac{1}{6}-4 \sum_{n=1}^{\infty} \frac{n q^{2 n}}{1-q^{2 n}}\right)$ and $q=e^{\sqrt{-1} \tau}$.
} 
Lemma 1 The R-matrix given in 2.4) and (2.5) satisfies

$$
R(w)=M \times P_{2}^{(+)},
$$

where $M$ is a non-degenerate matrix and $P_{2}^{(+)}$is the two-body symmetrization operator given by $P_{2}^{(+)}=\frac{1}{2}\left(1+P_{12}\right)$ in terms of the permutation operator $P_{12}: P_{12}\left(e_{i} \otimes e_{j}\right)=e_{j} \otimes e_{i}$.

This lemma tells that

$$
\operatorname{Ker}(R(w))=\text { the antisymmetric subspace of } \mathbb{C}^{n} \otimes \mathbb{C}^{n} \text {. }
$$

The above property enables us to construct symmetric fusion of the R-matrix in higher tensor space 14 and the intertwining vector in section 4.

Definition 1 The algebra of monodromy matrices $\mathcal{A}$ is an associative algebra generated by the matrix elements of the $L$-operator $L_{i j}(u), i, j=1, \cdots, n$ with the defining relations (1.1).

One can introduce a natural coproduct $\Delta: \mathcal{A} \rightarrow \mathcal{A} \otimes \mathcal{A}$ and counit $\epsilon: \mathcal{A} \rightarrow \mathbb{C}$

$$
\Delta\left(L_{i j}(u)\right)=\sum_{k} L_{i k}(u) \otimes L_{k j}(u), \quad \epsilon\left(L_{i j}\right)=\delta_{i j}
$$

making $\mathcal{A}$ a bi-algebra. Certain realization of the algebra $\mathcal{A}$ can be expressed in terms of the $\mathbb{Z}_{n}$ Sklyanin algebra [4, 5] which is an elliptical generalization of quantum group $U_{q}\left(g l_{n}\right)$.

In this paper, we investigate the center of the algebra $\mathcal{A}$, i.e., the set of the elements commuting with all the elements of $\mathcal{A}$. For a generic value of the crossing parameter $w$, the so-called quantum determinant of the L-operator $\left(\operatorname{det}_{q} L(u)\right)$ generates the center of the algebra $\mathcal{A}$ [15. However, it is known [10] that $\mathcal{A}$ for the eight-vertex model case $(n=2)$ the situation is quite different when $w=\frac{2}{N}, N$ is a positive odd integer. In this case, in addition to the "quantum determinant" $\operatorname{det}_{q} L(u)$, there exist much more central elements in $\mathcal{A}$. For the generic case of $n \geqslant 2$, the same phenomenon has been observed for $\mathcal{A}$ in the trigonometric limit [7, 8]. We shall study the extra central elements of $\mathcal{A}$ with a generic $n \geqslant 2$ in the "roots of unity" case: $w=\frac{n}{N}$, where $N$ and $n$ are positive coprime integers. Hereafter, we restrict the crossing parameter to the "roots of unity" case $w=\frac{n}{N}$, unless otherwise stated. 


\section{$3 \quad A_{n-1}^{(1)}$ Face Model and Face-Vertex Correspondence}

In this section, we formulate the fundamental Boltzmann weights of the $A_{n-1}$ type SOS face model and give the face-vertex correspondence relation.

An ordered pair $(a, b)\left(a, b \in \mathbb{C}^{n}\right)$ is called admissible if

$$
b-a=e_{i}, \quad i=1, \cdots, n,
$$

and denoted by $a \stackrel{1}{\rightarrow} b$. A path $p=\left(a^{(0)}, a^{(1)}, \cdots, a^{(l)}\right) \in\left(\mathbb{C}^{n}\right)^{l+1}$ is called an $l$-path from $a$ to $b$ if $a^{(0)}=a, a^{(l)}=b$, and all pairs $\left(a^{(i-1)}, a^{(i)}\right)$ for $i=1, \cdots, l$ are admissible.

Definition 2 An ordered pair $(a, b)\left(a, b \in \mathbb{C}^{n}\right)$ is called l-admissible if there exists an l-path from a to $b$, and denoted by $a \stackrel{l}{\rightarrow} b$.

Let $W\left(\begin{array}{cc|c}a & b & u \\ c & d & u\end{array}\right)$ denote the Boltzmann weight corresponding to a configuration $a, b, d$, $c \in \mathbb{C}^{n}$ around a face, ordered clockwise from the NW corner. We call that $W\left(\begin{array}{ll|l}a & b & u \\ c & d\end{array}\right)$ is admissible if $(a, b),(b, d),(a, c),(c, d)$ are all admissible. By definition non-admissible weights are set to 0 . The non-vanishing weights are given as follows

$$
\begin{aligned}
& W\left(\begin{array}{ll}
a & a+e_{\mu} \\
a+e_{\mu} & a+2 e_{\mu}
\end{array} \mid u\right)=\frac{\sigma(u+w)}{\sigma(w)}, \\
& W\left(\begin{array}{ll}
a & a+e_{\mu} \\
a+e_{\mu} & a+e_{\mu}+e_{\nu}
\end{array} \mid u\right)=\frac{\sigma\left(a_{\mu \nu} w-u\right)}{\sigma\left(a_{\mu \nu} w\right)}, \mu \neq \nu, \\
& W\left(\begin{array}{ll}
a & a+e_{\nu} \\
a+e_{\mu} & a+e_{\mu}+e_{\nu}
\end{array} \mid u\right)=\frac{\sigma(u) \sigma\left(a_{\mu \nu} w+w\right)}{\sigma(w) \sigma\left(a_{\mu \nu} w\right)}, \quad \mu \neq \nu, \\
& a_{\mu \nu}=\left\langle a, e_{\mu}-e_{\nu}\right\rangle, \quad \mu, \nu=1, \cdots, n .
\end{aligned}
$$

The weights given in (3.2)-(3.4) satisfy the star-triangle relation (or dynamical Yang-Baxter equation) [13]

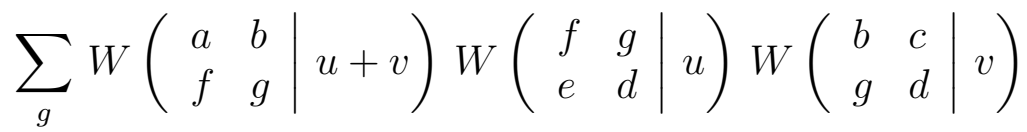

$$
\begin{aligned}
& =\sum_{g} W\left(\begin{array}{cc|}
a & g \\
f & e
\end{array} \mid v\right) W\left(\begin{array}{cc|c}
a & b & u \\
g & c & u
\end{array}\right) W\left(\begin{array}{cc|c}
g & c \\
e & d
\end{array} \mid u+v\right) .
\end{aligned}
$$

For a generic $\lambda \in \mathbb{C}^{n}$, define

$$
\begin{aligned}
& \lambda_{i}=\left\langle\lambda, e_{i}\right\rangle, \quad \bar{\lambda}_{i}=\left\langle\lambda, \bar{e}_{i}\right\rangle, \quad \bar{e}_{i}=e_{i}-\frac{1}{n} \sum_{k} e_{k}, \\
& \lambda_{i j}=\lambda_{i}-\lambda_{j}=\bar{\lambda}_{i}-\bar{\lambda}_{j}, \quad i, j=1, \cdots, n
\end{aligned}
$$


Let us introduce an intertwining vector-an $n$-component column vector $\phi_{\lambda, \lambda+e_{j}}(u)$ whose $k$-th element is

$$
\phi_{\lambda, \lambda+e_{j}}^{(k)}(u)=\bar{\theta}^{(k)}\left(u-n w \bar{\lambda}_{j}\right) .
$$

One can easily verify the following properties of the intertwining vector from the definition (3.9) and equation (3.7).

Lemma 2 The intertwining vector defined by (3.9) satisfies the following relation

$$
\begin{aligned}
& \phi_{\lambda+e_{j}, \lambda+e_{i}+e_{j}}(u)=\phi_{\lambda, \lambda+e_{i}}\left(u+w-n \delta_{i j} w\right), \quad i, j=1, \cdots, n, \\
& \phi_{\lambda, \lambda+e_{i}}(u+n)=\phi_{\lambda, \lambda+e_{i}}(u) .
\end{aligned}
$$

The intertwining vector (3.9) is different from that of Jimbo et al [13] by the definition of the $\bar{\theta}$-function given in (2.3) . The present definition is preferred because of its periodicity (3.11) which will play an important role in section 5. From the definition of the Boltzmann weights (3.2)-(3.4) and using the face-vertex correspondence obtained in [13], we have the following relation.

Theorem 1 The intertwining vector satisfies

$$
\begin{aligned}
& R_{12}(u-v) \phi_{\lambda, \lambda+e_{i}}(u) \otimes \phi_{\lambda+e_{i}, \lambda+e_{i}+e_{j}}(v) \\
& \quad=\sum_{k} W\left(\begin{array}{ll}
\lambda & \lambda+e_{k} \\
\lambda+e_{i} & \lambda+e_{i}+e_{j}
\end{array} \mid u-v\right) \phi_{\lambda+e_{k}, \lambda+e_{i}+e_{j}}(u) \otimes \phi_{\lambda, \lambda+e_{k}}(v) .
\end{aligned}
$$

Then the Yang-Baxter equation of the $\mathbb{Z}_{n}$ R-matrix $R(u)(1.2)$ is equivalent to the startriangle relation (3.6).

\section{Fusion Procedure}

The fusion procedure of the R-matrix and the intertwining vector is essential in handling the central elements of the algebra $\mathcal{A}$.

\subsection{The generic $w$ case}

In this subsection, we consider the case that the crossing parameter $w$ takes a generic value. Let us introduce operators $\mathcal{L}_{1 \cdots N}(u)$ and $\mathcal{R}_{1 \cdots N}(u)$ by

$$
\begin{aligned}
& \mathcal{R}_{1 \cdots N}(u) \equiv \mathcal{R}_{1 \cdots N ; 0}(u)=R_{10}(u+(N-1) w) \cdots R_{N 0}(u), \\
& \mathcal{L}_{1 \cdots N}(u)=L_{1}(u+(N-1) w) \cdots L_{N}(u) .
\end{aligned}
$$


We consider $\mathcal{L}_{1 \cdots N}(u)\left(\mathcal{R}_{1 \cdots N}(u)\right)$ as an operator acting on the so-called "auxiliary space" $V_{1} \otimes \cdots \otimes V_{N}, V_{i}=\mathbb{C}^{n}$ with the entries belonging to $\mathcal{A}$ (with the entries of the operator $\mathcal{R}_{1 \cdots N}(u)$ acting on an additional $n$-dimensional space denoted by 0 -th tensor space). Define an operator $S_{N}$ acting on $V_{1} \otimes \cdots \otimes V_{N}$ as follow

$$
S_{N}=\prod_{i=1}^{N-1} \prod_{j=i+1}^{N} R_{i j}((j-i) w),
$$

where both indices grow from left to right. For example, if $N=3$, then

$$
S_{3}=R_{12}(w) R_{13}(2 w) R_{23}(w) .
$$

Proposition $1 \mathrm{Ker}\left(S_{N}\right)$ is an invariant subspace in $V_{1} \otimes \cdots \otimes V_{N}$ of the operators $\mathcal{L}_{1 \cdots N}(u)$ and $\mathcal{R}_{1 \cdots N}(u)$.

Proof. Using the Yang-Baxter equation (1.2) and $R L L$ relation (1.1), one can derive

$$
\begin{aligned}
& S_{N} \mathcal{R}_{1 \cdots N}(u)=\mathcal{R}_{N \cdots 1}(u) S_{N}, \\
& S_{N} \mathcal{L}_{1 \cdots N}(u)=\mathcal{L}_{N \cdots 1}(u) S_{N},
\end{aligned}
$$

where $\mathcal{R}_{N \cdots 1}(u)$ and $\mathcal{L}_{N \cdots 1}(u)$ differ from $\mathcal{R}_{1 \cdots N}(u)$ and $\mathcal{L}_{1 \cdots N}(u)$ by permutation of all factors to the opposite order. Hence, $\mathcal{R}_{1 \cdots N}(u) \operatorname{Ker}\left(S_{N}\right) \subset \operatorname{Ker}\left(S_{N}\right), \mathcal{L}_{1 \cdots N}(u) \operatorname{Ker}\left(S_{N}\right) \subset$ $\operatorname{Ker}\left(S_{N}\right)$.

Now, let us consider the subspace $\operatorname{Ker}\left(S_{N}\right)$. Define

$$
W_{0}=\Sigma_{i=1}^{N-1} \operatorname{Ker}\left(R_{i, i+1}(w)\right) \subset V_{1} \otimes \cdots \otimes V_{N} .
$$

Using the Yang-Baxter equation (1.2), one can show that the factors of $S_{N}$ can be permuted into the order such that $R_{i, i+1}(w)$ for a given $i=1, \cdots, N-1$ is in the rightmost position. For example, if $N=3$ and $i=1$,

$$
S_{3}=R_{12}(w) R_{13}(2 w) R_{23}(w)=R_{23}(w) R_{13}(2 w) R_{12}(w) .
$$

So, $\operatorname{Ker}\left(S_{N}\right) \supset W_{0}$. Comparing the dimensions of the subspaces in the trigonometric limit, one can derive that $\operatorname{Ker}\left(S_{N}\right)=W_{0}$ for the generic crossing parameter $w$. However, when the crossing parameter $w$ is in the "roots of unity" case $w=\frac{n}{N}$, the subspace $\operatorname{Ker}\left(S_{N}\right)$ will be larger than $W_{0}$. 
It follows from Proposition 1 that $W_{0}$ is invariant under $\mathcal{L}_{1 \cdots N}(u)$ and $\mathcal{R}_{1 \cdots N}(u)$. Thus these operators can be defined in the quotient space $W_{0}^{\perp}$ which is defined as follow

$$
W_{0}^{\perp}=V_{1} \otimes \cdots \otimes V_{N} / W_{0}
$$

This also enables one to construct the symmetric fusion of the R-matrix and the L-operator in higher tensor space [14]. Moreover, the property (2.7) and the definitions of $W_{0}(4.4)$ and $W_{0}^{\perp}(4.5)$ imply that

$$
\begin{aligned}
& W_{0}^{\perp}=\text { the symmetric subspace of } V_{1} \otimes \cdots \otimes V_{N} \\
& \operatorname{dim}\left(W_{0}^{\perp}\right)=\frac{(N+n-1) !}{N !(n-1) !}, \quad \operatorname{dim}\left(W_{0}\right)=n^{N}-\frac{(N+n-1) !}{N !(n-1) !} .
\end{aligned}
$$

Define a complete symmetrizer $P_{N}^{(+)}$which is given in terms of the element of $N$-body permutation group denoted by $\mathcal{P}_{N}$ as follows:

$$
P_{N}^{(+)}=\frac{1}{N !}\left\{\sum_{P \in \mathcal{P}_{N}} P\right\}
$$

Proposition 1 and the equation (4.6) lead to the following fusion properties.

\section{Lemma 3}

$$
\begin{aligned}
& P_{N}^{(+)} \mathcal{R}_{1 \cdots N ; 0}(u)=P_{N}^{(+)} \mathcal{R}_{1 \cdots N ; 0}(u) P_{N}^{(+)} \\
& P_{N}^{(+)} \mathcal{L}_{1 \cdots N}(u)=P_{N}^{(+)} \mathcal{L}_{1 \cdots N}(u) P_{N}^{(+)}
\end{aligned}
$$

Hence, one can define the symmetric fused $R$-matrix $\mathcal{R}_{1 \cdots N}^{(s)}$ and $L$-operator $\mathcal{L}_{1 \cdots N}^{(s)}$ acting in $W_{0}^{\perp}$ as follows

$$
\begin{aligned}
& \mathcal{R}_{1 \cdots N}^{(s)}(u) \equiv \mathcal{R}_{1 \cdots N ; 0}^{(s)}(u)=P_{N}^{(+)} \mathcal{R}_{1 \cdots N ; 0}(u) P_{N}^{(+)} \\
& \mathcal{L}_{1 \cdots N}^{(s)}(u)=P_{N}^{(+)} \mathcal{L}_{1 \cdots N}(u) P_{N}^{(+)}
\end{aligned}
$$

For an $N$-admissible pair $(a, b)$, we choose one of its $N$-paths from $a$ to $b: p=(a, a+$ $\left.e_{i_{1}}, a+e_{i_{1}}+e_{i_{2}}, \cdots, a+\sum_{l=1}^{N} e_{i_{l}}\right), b=a+\sum_{l=1}^{N} e_{i_{l}}$. Let us introduce a fused intertwining vector $\Phi_{p ; a, b}(u)$ as follows

$$
\begin{aligned}
& \phi_{N ; a, b}^{i_{1}, \cdots, i_{N}}(u)=\phi_{a, a+e_{i_{1}}}(u+(N-1) w) \otimes \cdots \otimes \phi_{a+\sum_{l=1}^{N-1} e_{i_{l}}, a+\sum_{l=1}^{N} e_{i_{l}}}(u), \\
& \Phi_{p ; a, b}(u)=P_{N}^{(+)}\left\{\phi_{N ; a, b}^{i_{1}, \cdots, i_{N}}(u)\right\} .
\end{aligned}
$$

Noting Lemma 2], we obtain: 
Proposition 2 The fused intertwining vector given in (4.14) satisfies

$$
\Phi_{p ; a, b}(u)=\frac{1}{N !}\left\{P_{N}^{(+)}\left\{\sum_{P \in \mathcal{P}_{N}} \phi_{N ; a, b}^{i_{p_{1}}, \cdots, i_{p_{N}}}(u)\right\}\right\}
$$

where $P$ is an element of $\mathcal{P}_{N}$ :

$$
P=\left(\begin{array}{llll}
1 & 2 & \cdots & N \\
p_{1} & p_{2} & \cdots & p_{N}
\end{array}\right)
$$

The proof of this Proposition is relegated to Appendix.

Then, we have

Corollary 1 The fused intertwining vector given in (4.14) is independent of the choice of the $N$-path from a to $b$ for any $N$-admissible pair $(a, b)$.

Because of the path independence, we hereafter denote the fused intertwining vector given in (4.14) by $\Phi_{N ; a, b}(u)$ for any $N$-admissible pair $(a, b)$ instead of $\Phi_{p ; a, b}(u)$. From the construction of the fused intertwining vector, one easily has

$$
\operatorname{Span}\left\{\Phi_{N ; a, b}(u) \mid \text { for all } N \text {-admissible pairs }(a, b)\right\}=W_{0}^{\perp} \text {. }
$$

Now we establish an equivalence between the symmetric fused R-matrix (4.11) and Boltzmann weights considered in [16] through the fused intertwining vector.

Proposition 3 The fused intertwining vector satisfies the following fused face-vertex correspondence relation

$$
\begin{aligned}
& \mathcal{R}_{1 \cdots N ; 0}^{(s)}(u-v) \Phi_{N ; a, b}(u) \otimes \phi_{b, b+e_{j}}(v) \\
& \quad=\sum_{k} W_{N 1}\left(\begin{array}{cc}
a & a+e_{k} \\
b & b+e_{j}
\end{array} \mid u-v\right) \Phi_{N ; a+e_{k}, b+e_{j}}(u) \otimes \phi_{a, a+e_{k}}(v) .
\end{aligned}
$$

Here the fused Boltzmann weights $W_{N 1}\left(\begin{array}{ll|l}a & b & u \\ d & c & u\end{array}\right)$ are non-vanishing only for a configuration $a, b, c, d \in \mathbb{C}^{n}$ such that $a \stackrel{1}{\rightarrow} b, b \stackrel{N}{\rightarrow} c, a \stackrel{N}{\rightarrow} d, d \stackrel{1}{\rightarrow} c$ and the non-vanishing weights are given as follows

$$
W_{N 1}\left(\begin{array}{cc|}
a & b \\
d & c
\end{array} \mid u\right)=\left(\prod_{j=1}^{N-1} \frac{\sigma(u+j w)}{\sigma(w)}\right) \frac{\sigma\left(u+c_{\mu} w-b_{\nu} w\right) \prod_{\rho \neq \mu} \sigma\left(c_{\rho} w-b_{\nu} w+w\right)}{\prod_{\rho} \sigma\left(a_{\rho \nu} w+\delta_{\rho \nu} w\right)}
$$

when $b-a=e_{\nu}$ and $c-d=e_{\mu}$. 
Proof. Suppose $\left(a, a^{(1)}, \cdots, a^{(N-1)}, b\right)$ is an $N$-path from $a$ to $b$. By the fundamental facevertex correspondence relation of Theorem [1, one can derive

$$
\begin{aligned}
& \mathcal{R}_{1 \cdots N ; 0}(u-v)\left(\phi_{a, a^{(1)}}(u+(N-1) w) \otimes \cdots \otimes \phi_{a^{(N-1)}, b}(u)\right) \otimes \phi_{b, b+e_{j}}(v) \\
& =\sum_{a^{\prime(1)}, \cdots, a^{\prime}(N-1), b^{\prime}} W\left(\begin{array}{ll}
a & a^{\prime(1)} \\
a^{(1)} & a^{\prime(2)}
\end{array} \mid u-v+(N-1) w\right) W\left(\begin{array}{ll}
a^{(1)} & a^{\prime(2)} \\
a^{(2)} & a^{\prime(3)}
\end{array} \mid u-v+(N-2) w\right) \\
& \quad \times \cdots W\left(\begin{array}{ll}
a^{(N-2)} & a^{\prime(N-1)} \\
a^{(N-1)} & b^{\prime}
\end{array} \mid u-v+w\right) W\left(\begin{array}{ll}
a^{(N-1)} & b^{\prime} \\
b & b+e_{j}
\end{array} \mid u-v\right) \\
& \quad \times\left(\phi_{a^{\prime}(1), a^{\prime(2)}}(u+(N-1) w) \otimes \cdots \otimes \phi_{b^{\prime}, b+e_{j}}(u)\right) \otimes \phi_{a, a^{\prime}(1)}(v) .
\end{aligned}
$$

The definition of the fused intertwining vector (4.14) leads to

$$
\phi_{a, a^{(1)}}(u+(N-1) w) \otimes \cdots \otimes \phi_{a^{(N-1), b}}(u)=\Phi_{N ; a, b}(u) \bmod W_{0} .
$$

Multiplying equation (4.20) by $P_{N}^{(+)}$from the left and using Proposition 1, we have

$$
\begin{aligned}
& \text { LHS }=P_{N}^{(+)} \mathcal{R}_{1 \cdots N ; 0}(u-v)\left(\Phi_{N ; a, b}(u)+W_{0}\right) \otimes \phi_{b, b+e_{j}}(v) \\
& =\mathcal{R}_{1 \cdots N ; 0}^{(s)}(u-v) \Phi_{N ; a, b}(u) \otimes \phi_{b, b+e_{j}}(v), \\
& \operatorname{RHS}=\sum_{a^{\prime}(1)}\left\{\sum_{a^{\prime(2)}, \cdots, a^{\prime}(N-1), b^{\prime}} W\left(\begin{array}{ll}
a & a^{\prime(1)} \\
a^{(1)} & a^{\prime(2)}
\end{array} \mid u-v+(N-1) w\right) \cdots W\left(\begin{array}{ll}
a^{(N-1)} b^{\prime} \\
b & b+e_{j}
\end{array} \mid u-v\right)\right. \\
& \left.\times P_{N}^{(+)}\left\{\phi_{a^{\prime(1)}, a^{\prime(2)}}(u+(N-1) w) \otimes \cdots \otimes \phi_{b^{\prime}, b+e_{j}}(u)\right\}\right\} \otimes \phi_{a, a^{\prime}(1)}(v) \\
& =\sum_{a^{\prime}(1)}\left\{\sum_{a^{\prime(2)}, \cdots, a^{\prime}(N-1), b^{\prime}} W\left(\begin{array}{cc}
a & a^{\prime(1)} \\
a^{(1)} & a^{\prime(2)}
\end{array} \mid u-v+(N-1) w\right) \cdots\right.
\end{aligned}
$$

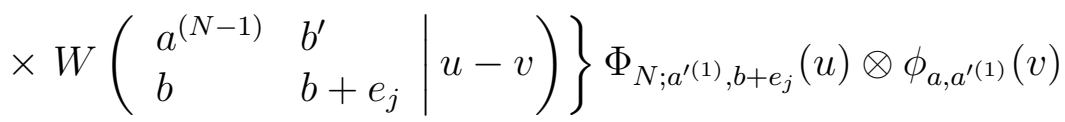

$$
\begin{aligned}
& =\sum_{a^{\prime(1)}} W_{N 1}\left(\begin{array}{ll}
a & a^{\prime(1)} \\
b & b+e_{j}
\end{array} \mid u-v\right) \Phi_{N ; a^{\prime(1)}, b+e_{j}}(u) \otimes \phi_{a, a^{\prime}(1)}(v) .
\end{aligned}
$$

We have used Corollary 1 in the second last equality and Lemma 2.2 of Ref.16] in the last equality. The expression of the fused Boltzmann weight $W_{N 1}\left(\begin{array}{ll}a & b \\ d & c\end{array} \mid u\right)$ was given in [16]. So, we complete the proof. 


\subsection{The "roots of unity" case}

From now on, we come back to the case that the crossing parameter takes value of "roots of unity" $w=\frac{n}{N}$. It was shown in [10] that for the eight-vertex (or $\mathbb{Z}_{2}$ ) case the subspace $\operatorname{Ker}\left(S_{N}\right)$ is larger than $W_{0}$ by an additional 2-dimensional subspace in "roots of unity" case. We shall show that $\operatorname{Ker}\left(S_{N}\right)$ is also larger than $W_{0}$ by an additional $n$-dimensional subspace for the generic $\mathbb{Z}_{n}$ case.

Let us introduce $n$ vectors $\Psi_{a}^{i}(u) \in V_{1} \otimes \cdots \otimes V_{N}, i=1, \cdots, n$,

$$
\Psi_{a}^{i}(u)=\Phi_{N ; a, a+N e_{i}}(u), \text { for a generic vector } a \in \mathbb{C}^{n}
$$

We denote the $n$-dimensional subspace spanned by the vectors $\left\{\Psi_{a}^{i}(u) \mid i=1, \cdots, n\right\}$ by $\bar{W}$ which is independent of the choice of $a$.

Proposition 4 When the crossing parameter $w=\frac{n}{N}, \operatorname{Ker}\left(S_{N}\right)=\bar{W} \oplus W_{0}$.

Proof. Using the Yang-Baxter equation (1.2), one can permute the order of the factors of $S_{N}$ in such way

$$
S_{N}=\cdots R_{1 N}((N-1) w) R_{1 N-1}((N-2) w) \cdots R_{12}(w) .
$$

Acting it on the vector $\phi_{N ; a, a+N e_{i}}^{i \cdots i}(u)$ defined in (4.13) (There exists a unique $N$-path for the $N$-admissible pair $\left(a, a+N e_{i}\right)$ such as $\left.\left(a, a+e_{i}, a+2 e_{i}, \cdots, a+N e_{i}\right)\right)$ and using Theorem 1. one can derive

$$
\begin{aligned}
S_{N} \phi_{N ; a, a+e_{i}}^{i \cdots i}(u)=\cdots R_{1 N}((N-1) w) R_{1 N-1}((N-2) w) \cdots R_{12}(w) \phi_{N ; a, a+N e_{i}}^{i \cdots i}(u) \\
=\cdots R_{1 N}((N-1) w) \cdots R_{12}(w) \phi_{a, a+e_{i}}(u+(N-1) w) \otimes \cdots \phi_{a+(N-1) e_{i}, a+N e_{i}}(u) \\
=\cdots \times \frac{\sigma(N w) \sigma((N-1) w) \cdots \sigma(w)}{(\sigma(w))^{N}} \phi_{a+(N-1) e_{i}, a+N e_{i}}(u+(N-1) w) \\
\quad \otimes \phi_{a, a+e_{i}}(u+(N-2) w) \otimes \cdots \otimes \phi_{a+(N-2) e_{i}, a+(N-1) e_{i}}(u) .
\end{aligned}
$$

The fact that $\phi_{N ; a, a+N e_{i}}^{i \cdots i}(u) \in \operatorname{Ker}\left(S_{N}\right)$ follows from the identity $\sigma(N w)=\sigma(n)=0$. Noting that

$$
\Psi_{a}^{i}(u)=\phi_{N ; a, a+N e_{i}}^{i \cdots i}(u) \bmod W_{0}
$$

and the fact $W_{0} \subset \operatorname{Ker}\left(S_{N}\right)$, one can show $\bar{W} \subset \operatorname{Ker}\left(S_{N}\right)$. Evidently $\bar{W} \cap W_{0}=\{0\}$. It follows that $\bar{W} \oplus W_{0} \subset \operatorname{Ker}\left(S_{N}\right)$. Then it remains to prove that

$$
\operatorname{dim} \operatorname{Ker}\left(S_{N}\right)=\operatorname{dim} W_{0}+\operatorname{dim} \bar{W} .
$$


However, the dimensions of these subspaces do not depend on the modulus $\tau$ of the torus. Taking the trigonometric limit $\tau \longrightarrow+\sqrt{-1} \infty$, the equation (4.26) has been already proved in 8 . So we complete the proof.

Proposition 1 and 4 show that $\bar{W}$ is invariant under the fused R-matrix $\mathcal{R}_{1 \cdots N}^{(s)}(u)$ defined in (4.11) and the L-operator $\mathcal{L}_{1 \cdots N}^{(s)}(u)$ defined in (4.12). Let $\hat{R}(u) \in \operatorname{End}\left(\bar{W} \otimes \mathbb{C}^{n}\right)$ and $\Lambda(u) \in \operatorname{End}(\bar{W}) \otimes \mathcal{A}$ be the restrictions of the fused operators $\mathcal{R}_{1 \cdots N}^{(s)}(u)$ and $\mathcal{L}_{1 \cdots N}^{(s)}(u)$ on $\bar{W}$, explicitly,

$$
\begin{aligned}
& \mathcal{R}_{1 \cdots N}^{(s)}(u) \Psi_{a}^{i}(u)=\hat{R}(u) \Psi_{a}^{i}(u)=\sum_{j=1}^{n} \hat{R}_{j}^{i}(u) \Psi_{a}^{j}(u), \quad \hat{R}_{j}^{i}(u) \in \operatorname{End}\left(\mathbb{C}^{n}\right), \\
& \mathcal{L}_{1 \cdots N}^{(s)}(u) \Psi_{a}^{i}(u)=\Lambda(u) \Psi_{a}^{i}(u)=\sum_{j=1}^{n} \Lambda_{j}^{i}(u) \Psi_{a}^{j}(u), \quad \Lambda_{i}^{j}(u) \in \mathcal{A} .
\end{aligned}
$$

\section{The Center}

Let us compute some of the fused Boltzmann weights with a specially chosen configuration $a, b, c, d \in \mathbb{C}^{n}$. By using Proposition [3 and the identity $\sigma\left(N w \delta_{i \nu}\right)=\sigma\left(n \delta_{i \nu}\right)=0$, we can derive for the crossing parameter $w=\frac{n}{N}$

$$
\begin{aligned}
W_{N 1} & \left(\begin{array}{ll}
a & a+e_{\nu} \\
a+N e_{i} & a+N e_{i}+e_{\mu}
\end{array} \mid u\right) \\
= & \left(\prod_{j=1}^{N-1} \frac{\sigma(u+j w)}{\sigma(w)}\right) \frac{\sigma\left(u+N w \delta_{i \mu}\right) \prod_{\rho \neq \mu} \sigma\left(a_{\rho \mu} w+N w \delta_{i \rho}\right)}{\prod_{\rho} \sigma\left(a_{\rho \mu} w+\delta_{\rho \mu} w\right)} \delta_{\mu \nu}
\end{aligned}
$$

in which the quasi-periodicity of the $\sigma$-function

$$
\sigma(u+N w)=\sigma(u+n)=(-1)^{n} \sigma(u), \quad N w=n,
$$

is used. This leads to a simple expression of the fused Boltzmann weight

$$
W_{N 1}\left(\begin{array}{ll}
a & a+e_{\nu} \\
a+N e_{i} & a+N e_{i}+e_{\mu}
\end{array} \mid u\right)=\left(\prod_{j=1}^{N} \frac{\sigma(u+j w)}{\sigma(w)}\right) \delta_{\mu \nu} .
$$

In order to compute the restricted R-matrix $\hat{R}(u)$ defined in (4.27), we evaluate the action of the fused R-matrix $\mathcal{R}_{1 \cdots N}^{(s)}$ defined in (4.11) on the vector $\Psi_{a}^{i}$

$$
\begin{aligned}
& \mathcal{R}_{1 \cdots N ; 0}^{(s)}(u-v) \Psi_{a}^{i}(u) \otimes \phi_{a+N e_{i}, a+N e_{i}+e_{j}}(v) \\
& \quad=\left(\prod_{k=1}^{N} \frac{\sigma(u-v+k w)}{\sigma(w)}\right) \Psi_{a+e_{j}}^{i}(u) \otimes \phi_{a, a+e_{j}}(v) .
\end{aligned}
$$


Using the shift property of the fundamental intertwining vector $\phi_{\lambda, \lambda+e_{i}}(u)$ (3.10), one can write the fused intertwining vector explicitly as

$$
\begin{gathered}
\Psi_{a}^{i}(u)=P_{N}^{(+)}\left\{\phi_{a, a+e_{i}}(u+(N-1) w) \otimes \phi_{a, a+e_{i}}(u+(N-1) w-n w)\right. \\
\left.\otimes \cdots \otimes \phi_{a, a+e_{i}}(u+(N-1) w-(N-1) n w)\right\} .
\end{gathered}
$$

The periodicity (3.11) enables one to further derive the following relation

$$
\phi_{a, a+e_{j}}(u+N w)=\phi_{a, a+e_{j}}(u+n)=\phi_{a, a+e_{j}}(u)
$$

The above equation and (5.4) imply the following relations

$$
\begin{aligned}
& \Psi_{a}^{i}(u+n w)=\Psi_{a}^{i}(u), \\
& \Psi_{a+e_{j}}^{i}(u)=\Psi_{a}^{i}(u+w) .
\end{aligned}
$$

Then

Proposition 5 When the crossing parameter is in "roots of unity" case $w=\frac{n}{N}$, the vector $\Psi_{a}^{i}(u)$ satisfies the following relation

$$
\Psi_{a+e_{j}}^{i}(u)=\Psi_{a}^{i}(u+w)=\Psi_{a}^{i}(u)
$$

Proof. The first equality is just equation (5.7). We are to prove the second equality $\Psi_{a}^{i}(u+$ $w)=\Psi_{a}^{i}(u)$. The expression of $\Psi_{a}^{i}(u)$ given in (5.4) and equation (5.5) imply that

$$
\Psi_{a}^{i}(u+N w)=\Psi_{a}^{i}(u+n)=\Psi_{a}^{i}(u)
$$

Due to the fact that $N$ and $n$ are positive coprime integers, one can choose an integer $l$ such that

$$
1+l N=0 \bmod n .
$$

The above property and relations (5.6) and (5.9) allow one to derive that

$$
\Psi_{a}^{i}(u+w)=\Psi_{a}^{i}(u+w+l N w)=\Psi_{a}^{i}(u+(1+l N) w)=\Psi_{a}^{i}(u)
$$

Hence, we complete the proof.

Using Proposition [5, the action of the fused R-matrix $\mathcal{R}_{1 \cdots N}^{(s)}$ on the vector $\Psi_{a}^{i}$ can be given explicitly as 


\section{Proposition 6}

$$
\begin{aligned}
& \mathcal{R}_{1 \cdots N ; 0}^{(s)}(u-v) \Psi_{a}^{i}(u) \otimes \phi_{a+N e_{i}, a+N e_{i}+e_{j}}(v) \\
& \quad=\left(\prod_{k=1}^{N} \frac{\sigma(u-v+k w)}{\sigma(w)}\right) \Psi_{a}^{i}(u) \otimes \phi_{a, a+e_{j}}(v) .
\end{aligned}
$$

Now, we calculate the exchange relation among the fused L-operators $\left\{\Lambda_{k}^{l}(u) \mid k, l=\right.$ $1, \cdots, n\}$ and the generators of $\mathcal{A}\left\{L_{i}^{j}(u) \mid i, j=1, \cdots, n\right\}$. Firstly, one can derive the following relation by the "RLL" relation (1.1)

$$
R_{1 \cdots N ; 0}(u-v) L_{1 \cdots N}(u) L_{0}(v)=L_{0}(v) L_{1 \cdots N}(u) R_{1 \cdots N ; 0}(u-v) .
$$

Proposition 1 allows one to restrict the above equation on $W_{0}^{\perp}$, namely in terms of the fused R-matrix (4.11) and L-operator (4.12)

$$
\mathcal{R}_{1 \cdots N ; 0}^{(s)}(u-v) \mathcal{L}_{1 \cdots N}^{(s)}(u) L_{0}(v)=L_{0}(v) \mathcal{L}_{1 \cdots N}^{(s)}(u) \mathcal{R}_{1 \cdots N ; 0}^{(s)}(u-v) .
$$

Proposition 4 and the fact that $\bar{W} \subset W_{0}^{\perp}$ allow one further to restrict the equation on $\bar{W}$. Finally, we have our main result.

Theorem 2 When the crossing parameter is in "roots of unity" case $w=\frac{n}{N}$, the elements $\left\{\Lambda_{j}^{i}(u) \mid i, j=1, \cdots, n\right\}$ defined in 4.28) are the central elements of $\mathcal{A}$, namely,

$$
\left[\Lambda_{j}^{i}(u), L_{l}^{k}(v)\right]=0 .
$$

Proof. For a generic $a \in \mathbb{C}^{n}$, we can introduce an $n \times n$ matrix $A$ with matrix elements $A_{j}^{i}$ :

$$
A_{j}^{i}=\phi_{a, a+e_{j}}^{(i)}(u), \quad i, j=1, \cdots, n .
$$

One can verify that $\operatorname{det}(A) \neq 0$. It means that

$$
\operatorname{Span}\left\{\phi_{a, a+e_{j}}(u) \mid j=1, \cdots, n\right\}=\mathbb{C}^{n} .
$$

This fact allows one to define matrix elements $\tilde{L}_{i}^{j}(u) \in \mathcal{A}$ from the L-operator $L(u)$ as follows

$$
L(u) \phi_{a, a+e_{i}}(u)=\sum_{j=1}^{n} \tilde{L}_{i}^{j}(u) \phi_{a, a+e_{j}}(u) .
$$

The conditions $w=\frac{n}{N}$ and (3.11) allow one to derive the following relation

$$
\phi_{a+N e_{i}, a+N e_{i}+e_{j}}(u)=\phi_{a, a+e_{j}}(u+N w)=\phi_{a, a+e_{j}}(u) .
$$


Acting both sides of equation (5.14) on the vector $\Psi_{a}^{l}(u) \otimes \phi_{a, a+e_{j}}(v)$ and using the definitions (4.28) and (5.17), we have

$$
\begin{aligned}
\mathrm{LHS} & =\sum_{k, i=1}^{n} \mathcal{R}_{1 \cdots N ; 0}^{(s)}(u-v) \Psi_{a}^{k}(u) \otimes \phi_{a, a+e_{i}}(v)\left\{\Lambda_{k}^{l}(u) \tilde{L}_{j}^{i}(v)\right\} \\
\mathrm{RHS} & =L_{0}(v) \mathcal{L}_{1 \cdots N}^{(s)}(v) \mathcal{R}_{1 \cdots N ; 0}^{(s)}(u-v) \Psi_{a}^{l}(u) \otimes \phi_{a, a+e_{j}}(v) .
\end{aligned}
$$

Proposition 6 and the relation (5.18) imply that we can further derive that

$$
\begin{aligned}
& \operatorname{LHS}=\left\{\prod_{k=1}^{N} \frac{\sigma(u-v+k w)}{\sigma(w)}\right\} \sum_{k, i=1}^{n} \Psi_{a}^{k}(u) \otimes \phi_{a, a+e_{i}}(v)\left\{\Lambda_{k}^{l}(u) \tilde{L}_{j}^{i}(v)\right\} \\
& \operatorname{RHS}=\left\{\prod_{k=1}^{N} \frac{\sigma(u-v+k w)}{\sigma(w)}\right\} \sum_{k, i=1}^{n} \Psi_{a}^{k}(u) \otimes \phi_{a, a+e_{i}}(v)\left\{\tilde{L}_{j}^{i}(v) \Lambda_{k}^{l}(u)\right\} .
\end{aligned}
$$

Hence $\left[\Lambda_{k}^{l}(u), \tilde{L}_{j}^{i}(v)\right]=0$. Equation (15.17) and non-degeneracy of the matrix $\left(A_{j}^{i}\right)$ enable us to derive that $\left[\Lambda_{k}^{l}(u), L_{j}^{i}(v)\right]=0$. Therefore, we complete the proof.

\section{Conclusions}

In this paper we have investigated the extra central elements (differing from the so-called quantum determinant $\left.\operatorname{det}_{q}(L(u))\right)$ of the algebra $\mathcal{A}$ of monodromy matrices associated with the $\mathbb{Z}_{n}$ R-matrix when the crossing parameter takes special value $w=\frac{n}{N}$. In the trigonometric limit, this case corresponds to the quantum group $U_{q}\left(g l_{n}\right)$ at roots of unity. Our results show that in even elliptic case the center of $\mathcal{A}$ is also extended and is generated by $n^{2}$ elements $\left\{\Lambda_{j}^{i}(u)\right\}$ in addition to $\operatorname{det}_{q}(L(u))$. For the special case of $n=2$, our result recovers that of [10].

\section{Acknowledgements}

W.-L. Yang is supported from the Japan Society for the Promotion of Science and Australian Research Council. A. Belavin is partially supported by grant RFBR-04-02-16027 and NATO grant PST.CLG.979008. He also would like to thank Yukawa Institute for Theoretical Physics, Kyoto University and Professor R. Sasaki for kind hospitality during his visit to Kyoto. 


\section{Appendix: Proof of Proposition 2}

The property (3.10) allows one to derive the following relation

$$
\phi_{\lambda+e_{j}, \lambda+e_{i}+e_{j}}(u)=\phi_{\lambda, \lambda+e_{i}}(u+w), \text { if } i \neq j .
$$

For a vector $B \in V_{1} \otimes \cdots \otimes V_{m}$, the corresponding symmetrized vector $B^{(s)}$ is given as

$$
B^{(s)}=P_{m}^{(+)}(B)=\frac{1}{m !}\left\{\sum_{P \in \mathcal{P}_{m}} P(B)\right\} .
$$

The terms obtained by non-trivial permutations of $B$ are called descendants. It is easy to show that the symmetrized vector satisfies

$$
B^{(s)}=P_{m}^{(+)}(P B), \quad \forall P \in \mathcal{P}_{m}
$$

Hereafter we shall always keep equations (A.1) and (A.2) in mind, because they will play an important role to proof the proposition.

Let us denote the vector $\phi_{m ; a, a+\sum_{l=1}^{m} e_{i_{l}}}^{i_{1} \cdots i_{m}}$ by $i_{1} \cdots i_{m}$, and the set of $\left\{\phi_{m ; a, a+\sum_{l=1}^{m} e_{i_{l}}}^{i_{p_{1} \cdots i_{p_{m}}}} \mid P \in \mathcal{P}_{m}\right\}$ by $\overbrace{i_{1} \cdots i_{m}}$. We shall prove the proposition by induction.

- Using the relation (A.1), we can prove that the equation (4.15) holds for the case of $N=2$.

- Suppose that (4.15) holds for the case of $2<N$. We are to prove it is satisfied for $N+1$.

1. In the case of $i_{N+1} \notin\left\{i_{1}, \cdots, i_{N}\right\}$,

$$
\Phi_{N+1 ; a, a+\sum_{l=1}^{N+1} e_{i_{l}}}^{i_{1} \cdots i_{N+1}} \propto P_{N+1}^{(+)}\left\{\begin{array}{c}
\overbrace{i_{1} \cdots i_{N}} \\
\vdots
\end{array} i_{N+1}\right\},
$$

where $\vdots$ stands for the descendants. Using the induction hypothesis and (A.1) we can derive that (A.3) is equal to

$$
\begin{aligned}
P_{N+1}^{(+)} & \left\{\begin{array}{ccccc}
i_{1} \cdots i_{N} & i_{N+1} \\
\vdots & & i_{1} \cdots i_{N+1} & i_{N} \\
\vdots & i_{1} \cdots i_{N+1} & i_{N-1} \\
&
\end{array}\right. \\
& =P_{N+1}^{(+)}\{\overbrace{i_{1} \cdots i_{N+1}}\} .
\end{aligned}
$$

So, 4.15) holds for $N+1$. 
2. If $i_{N+1} \in\left\{i_{1}, \cdots, i_{N}\right\}$, without loss of generality, let us suppose that $i_{N+1}=i_{N}$. Let $\left[i_{N}\right]^{\perp}$ denote the set $\left\{i_{l} \neq i_{N} \mid l=1, \cdots N-1\right\}$. Let $M$ be the number of elements in the set. Let $\left[i_{N}\right]_{l}^{\perp}, l=1, \cdots, M$, denote the element of the set. Similarly to the first case, we have

$$
\begin{aligned}
& \Phi_{N+1 ; a, a+\sum_{l=1}^{N+1} e_{i_{l}}}^{i_{1} \cdots i_{N} i_{N}} \propto P_{N+1}^{(+)}\left\{\begin{array}{c}
\overbrace{i_{1} \cdots i_{N}} \\
i_{N} \\
\vdots
\end{array}\right\}
\end{aligned}
$$

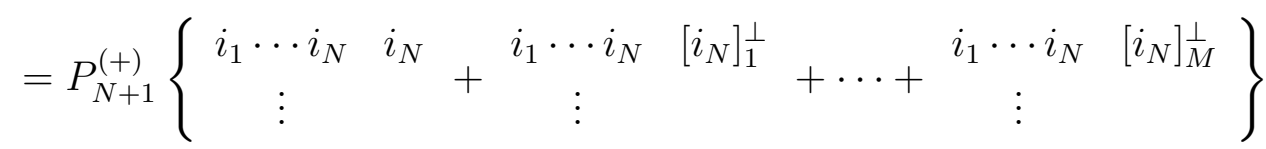

$$
\begin{aligned}
& =P_{N+1}^{(+)}\{\overbrace{i_{1} \cdots i_{N} i_{N}}\} \text {. }
\end{aligned}
$$

Then (4.15) holds for $N+1$.

Therefore (4.15) holds for any positive integer $N \geq 2$. Finally we complete the proof of Proposition 2$]$

\section{References}

[1] V. G. Drinfeld, Sov. Math. Dokl. 32 (1985), 254.

[2] V. G. Drinfeld, Proc. ICM, Berkeley (1986), 798.

[3] M. Jimbo, Lett. Math. Phys. 10 (1985), 63.

[4] E. K. Sklyanin, Func. Anal. Appl. 17 (1983), 273.

[5] B. Y. Hou and H. Wei, J. Math. Phys. 30 (1989), 2750.

[6] A. A. Belavin, Nucl. Phys. B 180 (1981), 189.

[7] C. De Concini, V. G. Kac, Colloque Dixmier (1989), 471.

[8] V. Tarasov, Commun. Math. Phys. 158 (1993), 459.

[9] R. J. Baxter, Exactly Solved Models in Statistical Mechanics, Academic Press, London, 1986.

[10] A. Belavin and M. Jimbo, Inter. J. Mod. Phys. A 19 (2004), 50. 
[11] E. T. Whittaker and G. N. Watson, A course of modern analysis: 4th edn., Cambridge University Press, Cambridge, 2002.

[12] M. P. Richey and C. A. Tracy, J. Stat. Phys. 42 (1986), 311.

[13] M. Jimbo, T. Miwa and M. Okado, Lett. Math. Phys. 14 (1987), 123; Nucl. Phys. B 300 (1988), 74.

[14] I. V. Cherednik, Funct. Anal. Appl. 19 (1985), 77.

[15] B. Y. Hou and Y.K. Zhou, J. Phys. A 23 (1990), 1147.

[16] M. Jimbo, A. Kuniba, T. Miwa and M. Okado, Commun. Math. Phys. 119 (1988), 543. 